\title{
Bacterial abundance and community composition in green, brown and red water from intensive catfish (Clarias sp.) culture ponds in Yogyakarta, Indonesia
}

\author{
KARUNIA ADETERA NUNGKI WIJAYANTI ${ }^{1}$, INDAH ISTIQOMAH ${ }^{2}$, MURWANTOKO ${ }^{2, v}$ \\ ${ }^{1}$ Fisheries Science Graduate Program, Department of Fisheries, Faculty of Agriculture, Universitas Gadjah Mada. Jl. Flora 1, Bulaksumur, Sleman55281, \\ Yogyakarta, Indonesia \\ ${ }^{2}$ Department of Fisheries, Faculty of Agriculture, Universitas Gadjah Mada. Jl. Flora 1, Bulaksumur, Sleman55281, Yogyakarta, Indonesia. Tel./fax.: \\ +62-274-563062, ‘email: murwantoko@ugm.ac.id
}

Manuscript received: 28 June 2021. Revision accepted: 13 August 2021.

\begin{abstract}
Wijayanti KAN, Istiqomah I, Murwantoko. 2021. Bacterial abundance and community composition in green, brown and red water from intensive Catfish (Clarias sp.) culture ponds in Yogyakarta, Indonesia. Biodiversitas 22: 3677-3684. Catfish (Clarias sp.) is an important aquaculture commodity in Indonesia and cultured in an intensive system. Microorganisms play an important role in maintaining water quality of aquaculture system. The objective of this study was to determine the bacterial abundance and community composition of green, brown and red water collected from intensive catfish culture ponds in Yogyakarta using next-generation sequencing method. The water samples were collected from intensive catfish culture ponds with different colors, namely green, brown and red ponds located in Yogyakarta. The DNA from water samples was extracted using DNA extraction kit and used as template for 16S rRNA amplification. The V3-V4 hypervariable regions of the 16S rRNA gene were amplified apply for next-generation sequencing technology. This study could explore effectively the bacterial community in water samples. The bacterial communities in this catfish culture water showed higher bacterial richness compared to the other aquaculture system. The diversity of the green, brown and red catfish culture water ponds was similar with the number OTUs of the green, brown and red water samples, which were $1269 ; 1387$ and 1323 OTUs respectively. The 694 OTUs (34.42\%) were common core microbiomes in all catfish culture ponds, the 212 OTUs (10.51\%) are present on green and brown water ponds, the 182 OTUs (9.02\%) were on green and red water ponds, and the 183 OTUs (9.07\%) were present on green and brown water ponds. However, the composition of the bacterial community was different. The most dominant phylum in green and brown water ponds was Proteobacteria with relative abundance in green water and brown water $71.6 \%$ and $47.0 \%$ respectively, whereas, the most dominant phylum in red water was Firmicutes (29.5\%). The dominance of Firmicutes phylum in red water ponds may be caused by application of probiotic bacteria, the high organic content, and low oxygen concentration.
\end{abstract}

Keywords: bacteria; composition; diversity; high-throughput sequencing, catfish culture

\section{INTRODUCTION}

The fisheries and aquaculture sector significantly expanded in the past few decades. Since the 1990s, a rise of $527 \%$ in global aquaculture production has been observed from 1990 to 2018. While capture fisheries production has been relatively stable, with some growth essentially concerning inland capture with a rise in global capture fisheries production from 1990 to 2018 is $14 \%$. World aquaculture production of farmed aquatic animals has been dominated by Asia, with $89 \%$ share in the last two decades with major producing countries is China, India, Indonesia, and Vietnam (FAO 2020).

Microorganisms play an important role in the aquaculture system. The microbial communities associated with the fish and their environment can improve water quality, reduce the abundance of fish pathogenic bacteria and ultimately improve fish survival (Benzton-Tilla et al. 2016). The importance of microbial diversity and its role in maintaining fish health in aquaculture systems has been increasingly recognized in recent years. However, there is still a major knowledge gap regarding the ecology, composition and dynamics of microbial plankton assemblages during fish production. Besides having direct effects on fish health and quality in aquaculture settings, microbial communities also influence fundamental processes such as nutrient cycling and water purification (Duarte et al. 2019). The healthy microbiome is mainly a resistant, resilient, and healthy functional core composed of a blend of articular microbial gene families, metabolic modules, and regulatory pathways capable of resisting any agitation due to internal or external factors (Rajeev et al. 2021).

Controlling the microorganisms associated with aquaculture systems (i.e. the aquaculture microbiome) has always been essential in high-intensity rearing of fish (Ditttman et al. 2017). The microbial-based systems represent one of the most viable strategies to achieve sustainable aquaculture. These systems are based on the promotion of microbial proliferation, either heterotrophic or autotrophic microorganisms. These microbes are expected to use, recycle and transform the excess of nutrients from feces, dead organisms, unconsumed food, and diverse metabolites into biomass, which the cultured organisms would further consume. (Martínez-Córdova 2014)

The implementation of microbiome-based products practiced by the specific functional groups of the 
aquaculture microbiome is enriched by adding, for example, carbon-rich substrates. These applications of targeted microbiome-based products containing a seeding microbial assemblage to aid the heterotrophic assimilation of inorganic nitrogen and/or the nitrification process are now a common practice in intensive tropical pond-based aquaculture systems (Ditttman et al. 2017). The ability of organisms to adapt to a particular habitat or living conditions mainly depends on their phenotypic plasticity. Therefore, the treatment approaches are now targeted towards modulating the aquatic species' microbiome and using them as beneficial partners to overcome the challenges confronted by the aquaculture sector (Rajeev et al. 2021).

Microbial communities in natural aquatic environments respond rapidly to changes in their immediate environment. These changes may be subtle and may manifest themselves as regulating certain metabolic pathways, or they may cause changes to the overall microbial community composition and functionality (Benzton-Tilla et al. 2016). The fundamental baseline information concerning the microbial dynamics of these systems and how ecological interactions can be used to modulate microbial assemblages (Duarte et al. 2019). The high-throughput sequencing (HTS) technologies have been applied as tool microbial ecology of these systems. The HTS has been used to characterize both the healthy and diseased aquaculture microbiome, to investigate the microbial community composition in recirculating aquaculture systems (BenztonTilla et al. 2016), investigated temporal variations of bacterioplankton and the seasonal dynamics of bacterial and microeukaryotic plankton communities e.g. in a semiintensive European seabass aquaculture system (Martins et al. 2018; Duarte et al. 2019). The seasonal studies showed that changes in environmental variables influence the overall microbial communities. (Roquigny et al. 2021).

Catfish is one of the important aquaculture commodities in Indonesia. In 2014, national catfish production was 679,379 tons which increased to $1,280,099$ tons in 2018 . This means that national catfish production from 2014 to 2018 increased by $88.4 \%$ (DJPB, 2020). The catfish have been intensively cultured in the Yogyakarta area. The farmers found the condition of water in ponds showed different colors. They believed that this different color gave different productivity. So, the microbiome from those different color ponds is important to be explored. The present study aimed to determine the bacterial abundance and community composition from green, brown and red water from intensive catfish culture ponds using the nextgeneration sequencing method. This is perhaps, the first study using high-throughput sequencing to analyze the bacterial community on freshwater aquaculture ponds in Indonesia.

\section{MATERIALS AND METHODS}

\section{Sample collection}

This research was conducted in intensive catfish (Clarias sp) culture ponds which have different colors, namely green, brown and red ponds in Sleman Regency of Yogyakarta Indonesia. The green water catfish culture pond is located in Kalasan District as a rectangular earthen pond with an area of $120 \mathrm{~m}^{2}$ with a stocking density of 60 fish $/ \mathrm{m}^{2}$. The brown water pond is located in Prambanan District as concrete wall construction with earthen bottom on a rectangular shape with an area of $30 \mathrm{~m}^{2}$ with a stocking density of $100 \mathrm{fish} / \mathrm{m}^{2}$. The red culture pond is located in Berbah as a round tarpaulin pond with an area of $7 \mathrm{~m}^{2}$ stocked with catfish at a density of $200 \mathrm{fish} / \mathrm{m}^{2}$. The water from the ponds was collected by taking one liter of water from the middle of the water column of pond. All the water samples were placed in a cool box, transported to the Fish Health and Environment Laboratory at Universitas Gadjah Mada (Yogyakarta, Indonesia), and stored in a refrigerator at $4^{\circ} \mathrm{C}$ for further analysis.

\section{DNA extraction}

Total $30 \mathrm{~mL}$ water samples were centrifuged at 3000xg for five minutes and settled pallets were then collected. The pellets were extracted their DNA using Genomic DNA Extraction Mini Kit (Favorprep ${ }^{\mathrm{TM}}$ Favorgen) following the manual protocol. The quality and concentration of the total DNA were estimated through electrophoresis on $1 \%$ agarose gel and NanoDrop MN-913A MaestroNano Pro (MaestroGen Inc., Hsinchu, Taiwan). The genomic DNA with a concentration of $50 \mathrm{ng} / \mu \mathrm{L}$ and DNA purity (A260/280) within the range of 1.8-2.0 were used for amplicon sequencing.

\section{Sequencing preparation and bioinformatics analysis}

Equal volumes of the total genomic DNA from green, brown and red water ponds proceeded for NGS. The V3V4 hypervariable regions of the $16 \mathrm{~S}$ rRNA gene were amplified using the forward primer $341 \mathrm{~F}\left(5^{\prime}-\right.$ CCTAYGGGRBGCASCAG-3') and the reverse primer 806R (5'-GGACTACNNGGGTATCTAAT-3') (Yu et al. 2005)

The DNA amplifications were performed with Phusion ${ }^{\circledR}$ High-Fidelity PCR Master Mix (New England Biolabs) in accordance with the manufacturer's instructions. The PCR products were then mixed with $1 \times$ loading buffer (containing SYB green) at the same volume, detected on $2 \%$ agarose gel, and purified with a GeneJETTM gel extraction kit (Thermo Fisher Scientific). Sequencing libraries were generated using Ion Plus Fragment Library Kit (Thermo Fisher Scientific) in accordance with the manufacturer's recommendations. Their quality was assessed with a Qubit 2.0 fluorometer (Thermo Fisher Scientific). The libraries were then sequenced on an Ion S5TM XL platform (Thermo Fisher Scientific), and $400 \mathrm{bp}$ to $600 \mathrm{bp}$ single-end reads were generated. This PCR amplification and amplicon sequencing were carried out by a service company (NovogeneAIT) in Singapore.

Single-end reads were further assigned to the samples based on their unique barcodes and truncated by cutting off the barcodes and primer sequences. Raw reads were filtered under specific filtering conditions to obtain highquality clean reads in accordance with the Cutadapt 
(V1.9.1) quality control process (Martin 2011). Then, chimera was removed by comparing the reads with the Silva 132 reference (Quast et al. 2013; https://www.arbsilva.de/) via the UCHIME algorithm (Edgar et al. 2011), and clean reads were finally obtained. Sequences with more than $97 \%$ similarity were clustered into the same operational taxonomic units (OTUs) by using the QIIME software version 1.9.1 (Caporaso et al. 2010) in accordance with the UCLUST method (Edgar 2010). The representative sequence of each OTU was screened for further annotation. Each representative sequence was then assigned using the Silva 132 reference based on the Mothur algorithm to annotate taxonomic information.

Alignment of the OTU representative sequences was performed using the MUSCLE software (version 3.8.31) to construct phylogenetic metrics such as Unifrac, which aimed to study phylogenetic relationship of different OTUs and the difference in the dominant species in various samples (Edgar 2004). Data normalization of OTUs abundance was done using a standard sequence number corresponding to the sample with the least sequences. This output normalized data were further used for rarefaction, rank abundance, bacterial community bar plot, bacterial community heatmap, principal coordinate analysis (PCoA), and Weighted Unifrac distance were determined and performed with QIIME (version 1.9.1) and then displayed with R software version 2.15.3 (Ihaka and Gentleman 1996). Alpha diversity is applied in analyzing complexity of species diversity for a sample through Chao1, Shannon, Simpson indices. All these indices in our samples were calculated with QIIME(Version 1.7.0) and displayed with R software (Version 2.15.3).

\section{RESULTS AND DISCUSSION}

\section{Sequence analysis}

High-throughput sequencing was performed to analyze the 16S rRNA gene of bacteria present in the different watercolor from pond of catfish (Clarias sp) culture. In the 16S rRNA gene sequencing, 440,433 effective sequences were obtained from three samples after chimera filtration. The number of effective sequences in the green, brown and red water samples were 145,588; 142,077 and 152,768 $(126,518 ; 121,109$ and 127,650$)$ respectively. The rarefaction curve which tended to reach flat curve (Figure 1a) indicated that the bacteria were effectively explored and only the scarce bacteria remained. The rarefaction curve of each sample revealed that the number of the observed species in equal, which is also supported by the rank abundance curve (Figure $1 \mathrm{~b}$ ).

All the effective sequences with $97 \%$ similarity were then grouped into the same, and this study could identify 2016 OTU. The number of OTUs was equal among three different color pond water, with the number OTUs of the green, brown and red water samples were 1269; 1387, and 1323 OTUs respectively. The bacterial community among three water samples was relatively similar, as indicated by the range or difference from the maximum-minimum value indexes are small/narrow. The bacterial/OTU richness as indicated by Chao1 were ranged from 1475.26 to 1331.71 . The evenness bacterial/OTU as indicated by Shannon index was ranged from 7.35 to 6.50 . Whereas the bacterial/OTU dominance by Simpson's index were ranged from 0.981 to 0.958 (Table 1).

Table 1. Alpha diversity indices of the bacteria from the green water pond (green12), brown water pond (brown23) and red water pond (red33).

\begin{tabular}{cccc}
\hline \multirow{2}{*}{ Sample } & \multicolumn{3}{c}{ Alpha diversity indices } \\
\cline { 2 - 4 } & Chao1 & Shannon & Simpson \\
\hline Green12 & 1331.71 & 6.50 & 0.958 \\
Brown23 & 1475.26 & 7.64 & 0.987 \\
Red33 & 1378.07 & 7.35 & 0.981 \\
\hline
\end{tabular}

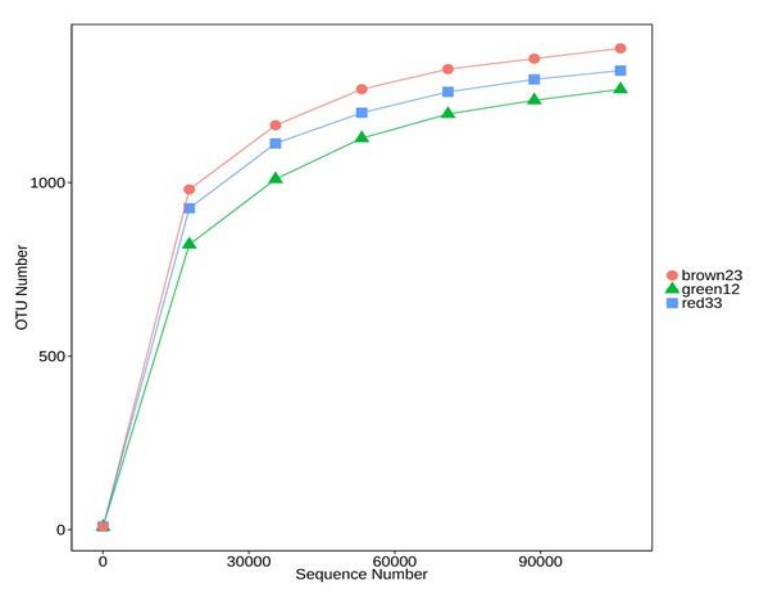

A

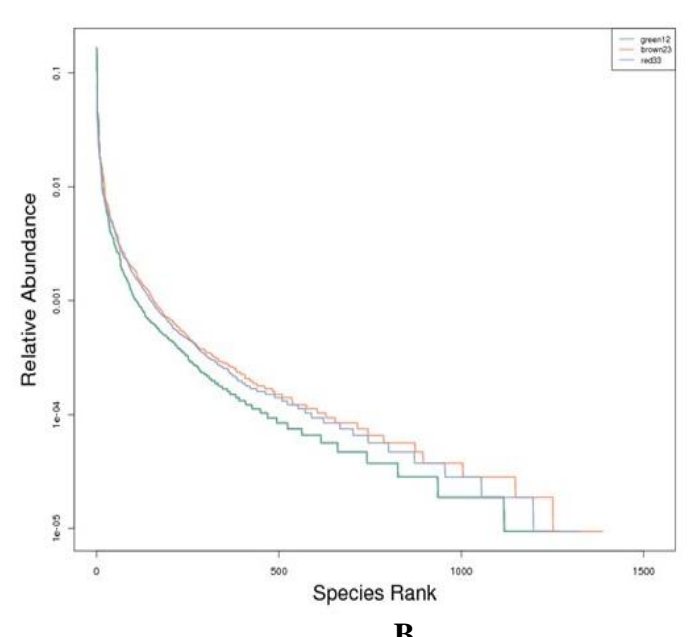

B

Figure 1. Rarefaction curves (A) and rank abundance curve (B) resulted from the 16S rRNA gene sequencing of the green (green12), brown (brown23) and red (red33) water pond 
Among 2016 of identified OTUs, the 694 OTUs $(34.42 \%)$ were common core microbiomes in the catfish culture pond as overlapped to all three color samples. The 212 OTUs $(10.51 \%)$ are present on green and brown water ponds, the 182 OTUs $(9.02 \%)$ are present on green and red water ponds, and the 183 OTUs $(9.07 \%)$ are present on green and brown water ponds. The rest OTUs were only present in the green, brown and red water samples were 181; 298 and 182 OTUs respectively (Figure 2).

\section{Bacterial diversity and abundance analysis}

All the sequences obtained from the three samples were assigned from phylum to genus levels. In particular, the three most dominant phyla were identified as Proteobacteria, Firmicutes and Bacteriocidetes possessed the relative abundance as $88.6 \%, 82.2 \%$, and $75.1 \%$ of the green, brown and red water respectively. The most dominant phylum was different among samples with the green water was dominated by Proteobacteria $(71.6 \%)$, Bacteriodetes $(10.6 \%)$, Firmicutes $(6.4 \%)$, brown water dominated by Proteobacteria $(47.0 \%)$, Bacteriodetes (24.1\%), Firmicutes $(11.0 \%)$, whereas red water dominated by Firmicutes (29.5\%), Proteobacteria (26.9\%), Bacteriodetes (18.6\%) (Figure 3).

In green water, the Betaproteobacteria was the most abundant class with the relative abundance of $41.9 \%$ followed by gammaproteobacteria with a relative abundance of $21.6 \%$. In brown water, the Betaproteobacteria was the most abundant class with the relative abundance of $15.9 \%$ followed by alphaproteobacteria with a relative abundance of $14.3 \%$ The Clostridia is most abundant class in redwater with relative abundance of $25.5 \%$ followed by bacteroidia with a relative abundance of $14.0 \%$ (Figure 4).

At the family level, the most abundant genus of the green, brown and red water was Rhodocyclaceae, Comamonadaceae, and Cristensenellaceae respectively. The Rhodocyclaceae was found in the green, brown and red water at relative abundance of $31.7 \%, 6.4 \%$, and $1.3 \%$ respectively. The Comamonadaceae and Saprospiraceae were found in significant amounts in the green and brown water. The Comamonadaceae was found at relative abundance of $7.7 \%$ and $6.3 \%$ on the brown and green water respectively, while Saprospiraceae was found at relative abundance of $5.5 \%$ and $5.5 \%$ respectively. The Methylococcaceae was found in red water found at relative abundance of $9.7 \%$ and in other water only found at relative abundance of less than $1 \%$ (Figure 4).

The heatmap analysis revealed that the bacterial community composition in the water ponds differed among different color water, as indicated by different dominant genera. The genera of Thiothrix, Heliscomenobacter, Peredibacter, Competibacter, Thaurea, Methyloparacoccus, Dechloromonas were found dominant in green water. The genera of Bacterriodes, Rhodobacter, Propionivibrio, Desulfomicrobium, Novosphingobium, and Acidmaniobacter were dominant in brown water. The genera of Paludibacter, Calidithrix, Microbacter, Christensenellaceae, Desulfobulbus were found dominant in redwater (Figure 5).

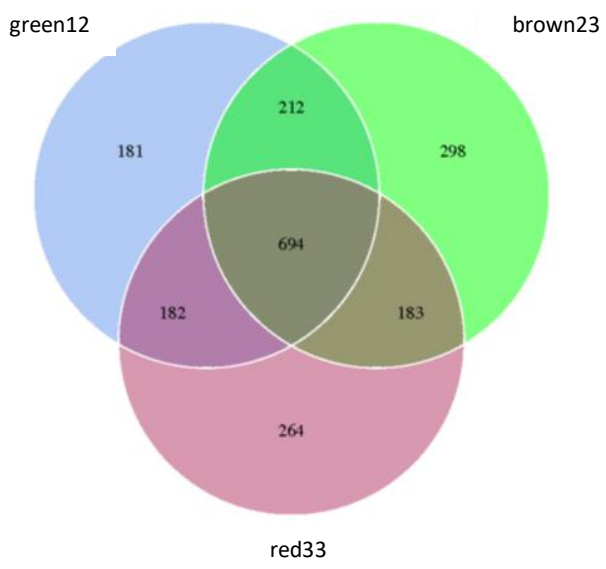

Figure 2. Venn diagram of the number of OTUs in the green water pond (green12), brown water pond (brown23), and red water pond (red33).

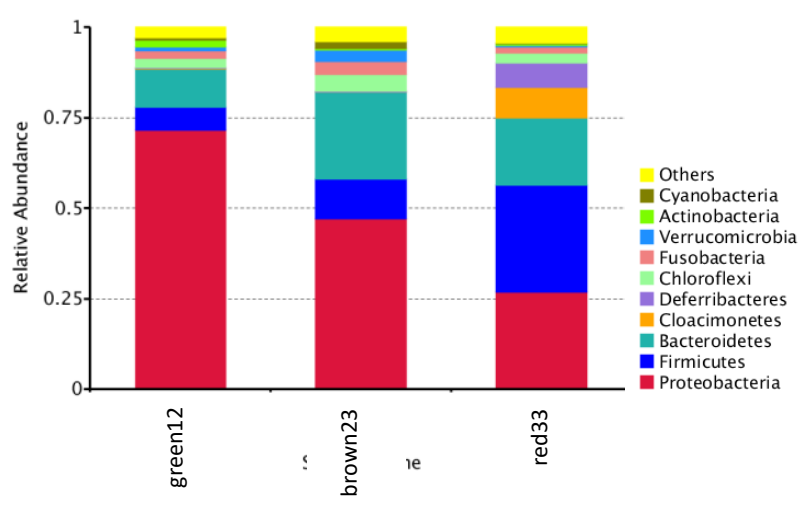

Figure 3. Relative abundance of bacterial communities at the phylum level of the green water pond (green12), brown water pond (brown23) and red water pond (red33)

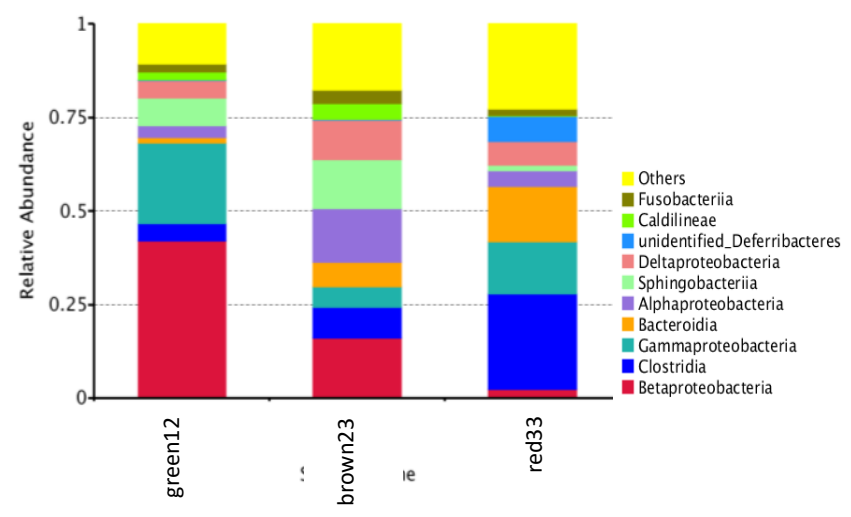

Figure 3. Relative abundance of bacterial communities at the class level of the green water pond (green12), brown water pond (brown23) and red water pond (red33) 


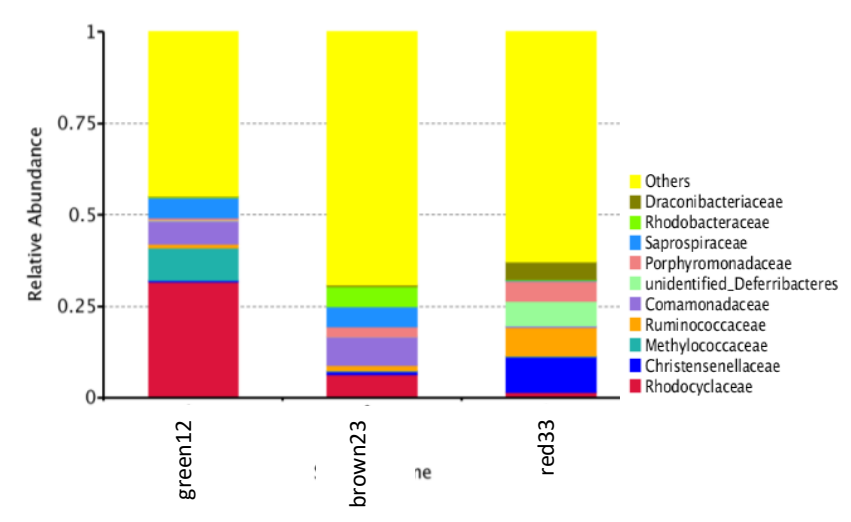

Figure 4. Relative abundance of bacterial communities at the ordo level of the green water pond (green12), brown water pond (brown23) and red water pond (red33)

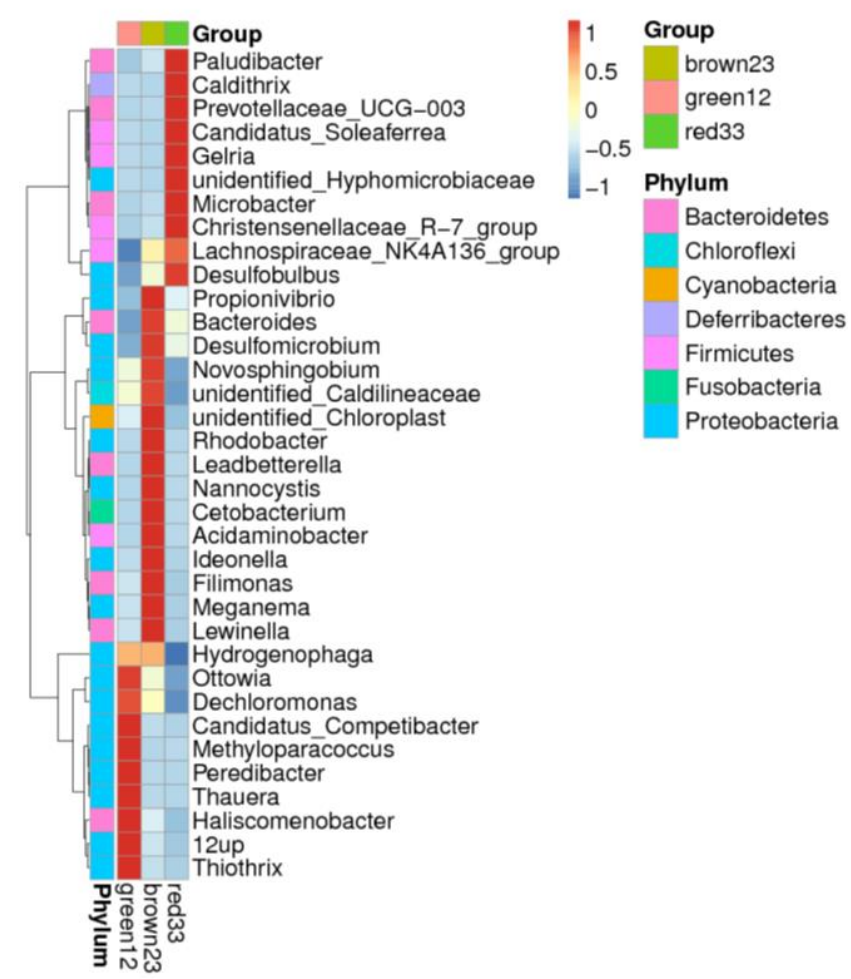

Figure 5. Abundance heatmap of bacterial communities at the genus level in the green water pond (green12), brown water pond (brown23) and red water pond (red33). The absolute value of the scale represents the distance between the raw score and the mean of the standard deviation. The value is negative when the raw score is below the mean, and vice versa

\section{Similarity analysis of bacterial composition}

The similarity between bacterial communities from different water was further analyzed through PCoA. The PCoA plot showed that the green, brown and red water were separated from each other (Figure 6). The relationship analysis using the unweighted pair group method with arithmetic mean (UPGMA) method showed that the green and brown water was closer than the red water (Figure 7).

\section{Discussion}

Microorganisms play important role in aquaculture system. The microbial communities can improve water quality, reduce the abundance of fish pathogenic bacteria, improve fish survival (Benzton-Tilla et al. 2016), and play fundamental processes such as nutrient cycling and water purification (Duarte et al 2019). Based on the study on the bacterial community structure and functional changes due to Chilean salmon aquaculture, the bacterial shifts can be used as indicators of aquaculture perturbations (Hornick and Buschmann 2018). However, there is still a major knowledge gap regarding the ecology, composition, and dynamics of microbial plankton assemblages during fish production (Duarte et al. 2019). The fish farmers in Yogyakarta have found different colors of water media of intensive catfish culture. They believed that this watercolor pond has different fish productivity. This study explores the bacterial communities from the green, brown and red water from catfish culture ponds using high throughput sequencing (HTS) or new generation sequencing (NGS) technology.

This study's bacterial communities in catfish culture water showed higher bacterial richness than the other aquaculture system. The bacterial richness as Chaol in this study was ranged from 1475.26 to 1331.71 (average 1394.9). In contrast, the bacterial community in pond of mina padi integrated culture was 1169.9 (Herlambang et al. 2021), the semi-intensive aquaculture for European sea bass (Dicentrarchus labrax) was ranged from 216.53 to 1148.86 (Duarte et al. 2019), the sediments from Chilean salmon aquaculture sites were ranged from 275.4 to 331.9 (Hornick and Buschmann 2018). The intensive catfish culture has put a lot of input such as the high nutrient of feed, so that the nutrient content of water is also high, giving possibility for many microorganisms to grow. The catfish culture in Yogyakarta is land-based aquaculture so that only limited water and nutrients flow out to the open system.

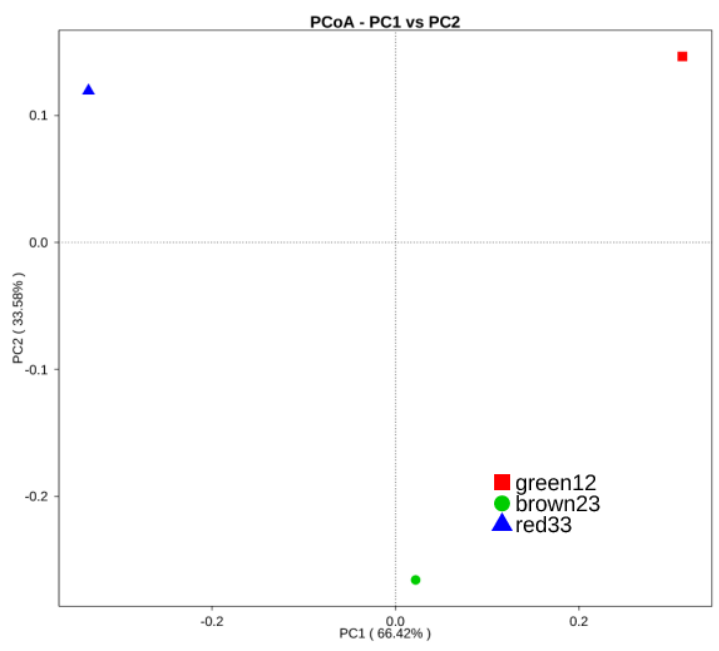

Figure 6. Principal coordinate analysis $(\mathrm{PCoA})$ of the green water pond (green 12), brown water pond (brown23), and red water pond (red33) based on Weighted Unifrac distance 


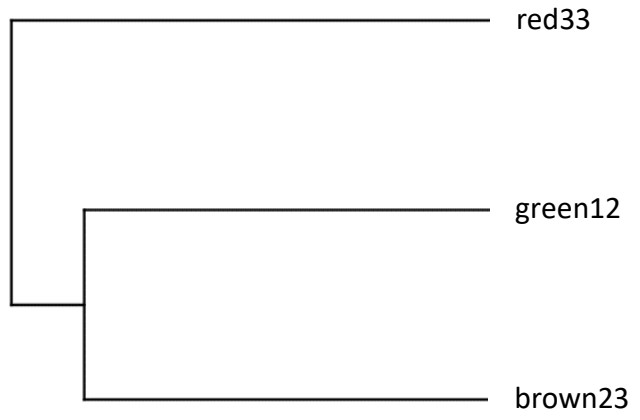

Figure 7. UPGMA The relationship of bacterial community analysis the green water pond (green12), brown water pond (brown23), and red water pond (red33) using UPGMA methods

The diversity of the green, brown, and red catfish culture water ponds were similar as indicated by the relatively similar Shannon index which ranged from 7.35 to 6.50. The dominance among those three color water ponds was also similar as indicated by Simpson's index, which ranged from 0.981 to 0.958 . However, the composition of the bacterial community on those three color water ponds was different. The most dominant phylum in green and brown water ponds was Proteobacteria with relative abundance in green water of $71.6 \%$ and in brown water of $47.0 \%$. Whereas the most dominant phylum in red water was Firmicutes $(29.5 \%)$. The Bacteriodetes were always second most dominant in green, brown, and red water ponds at relative abundance of $10.6 \%, 24.1 \%$, and $18.6 \%$ respectively. This study showed that the most dominant phylum in green and brown water samples was Proteobacteria. Other studies showed that Proteobacteria was also found as the most dominant phylum of bacterial communities in lakes (Kurilkina et al. 2016; Huang et al. 2017; Zhang et al. 2019), river ( $\mathrm{Li}$ et al. 2008; Abia et al. 2018), freshwater aquaculture (Fan et al. 2015; Qin et al. 2016), and minapadi field in India (Gupta 2020; Herlambang et al. 2021), mariculture of European sea bass (Martins et al. 2018; Duarte et al. 2019). Proteobacteria exhibits diverse biochemical energy-generating mechanisms; some of its members are phototrophs (e.g., purple Chromatium, Rhodospirillum), or chemolithotrophs (e.g., sulfur-oxidizing bacteria, including Thiobacilli, and ammonia-oxidizing bacteria, such as Nitrosomonas), chemoorganotrophs (e.g., Escherichia coli) (Kersters et al. 2006). Hence, many microbes belonging to Proteobacteria might play a crucial role in various biogeochemical cycles.

The red water of the red water pond of catfish culture in this study was dominated by Firmicutes phylum. The Firmicutes have been found as the most dominant phylum in shrimp samples from Lake Nokoue (Dadabe et al. 2016). The Firmicutes have also been found to be the most dominant in sugar cane processing plants characterized by high sugar/hemicellulose levels, available moisture, and warm conditions (Sharmin et al. 2013). It seems that the Firmicutes play a role in dynamic processes in the high organic matter content waters. The Firmicutes showed dramatic variation with incubation time during the decomposition Microcystis biomass process, whereas the other dominant phyla showed no discernable trends (Zao et al. 2017). The most dominant class of Firmicutes phylum in the red water pond was Clostridia. Clostridia are an extremely polyphyletic class of Firmicutes, lack aerobic respiration, obligate anaerobes, most of their species are Gram-positive. They can be found in decaying vegetation, marine sediment, soil, and intestinal tract of humans and other vertebrates. The spores of sulfite-reducing anaerobes (Clostridia) are widespread in the environment. The dominance of this Firmicutes phylum may be caused by application of probiotic bacteria, the high organic content, and low oxygen concentration in red water ponds.

At the class level, Betaproteobacteria was the most abundant in green and brown water at relative abundance of $41.9 \%$ and $15.9 \%$ respectively. The second abundant green and brown water class was different as the Gammaproteobacteria (21.6\%) and Alphaproteobacteria (14.3\%), respectively. The minapadi system's water study showed that those three classes as Betaproteobacteria, Alphaproteobacteria, and Gammaproteobacteria were the three most dominant classes (Herlambang et al. 2021). However, the other studies showed different dominant classes in several aquaculture systems. The Alphaproteobacteria $\quad(42.60 \pm 14.83 \%), \quad$ Flavobacteria $(23.70 \pm 10.04 \%)$, Gammaproteobacteria (15.06 $\pm 8.09 \%)$ were the most abundant classes a semi-intensive European seabass (Dicentrarchus labrax) aquaculture system in Ria de Aveiro estuarine lagoon, Portugal during 2012 (Martin et al. 2018). The sampling events throughout the year of 2014 from aquaculture system showed that the most abundant bacterial classes were Gammaproteobacteria (41.73 $\pm 4.71 \%), \quad$ Flavobacteriia $\quad(20.79 \pm 7.26 \%)$, Alphaproteobacteria, (13.55 $\pm 5.26 \%$ ) (Duarte 2019). The seasonal survey in a sea bass farm in France showed that Gammaproteobacteria, Alphaproteobacteria, and Deltaproteobacteria were the three main classes composing the Proteobacteria phylum with $55.88 \%, 37.20 \%$ and $6.82 \%$, respectively (Roquigny et al. 2020). Those results indicated that at class level, the bacterial composition was more dynamic.

Betaproteobacteria is a typical class of bacteria found in various freshwater habitats (Hahn 2006). The presence and abundance of Betaproteobacteria in freshwater are associated with its ability to respond quickly to changes in nutrient content, such as a rapid response to dissolved organic carbon (Newton et al. 2006). Several Alphaproteobacteria members are involved in the nitrogen cycle (Newton et al. 2011). Ecologically, Gammaproteobacteria helps to modulate excess nitrate (Fernandes et al. 2014).

The present study has effectively explored the bacterial community of the green, brown, and red water from catfish intensive culture ponds. Those three types of ponds have similar bacterial richness but different in bacterial composition from phyla level to genera level. The anthropogenic activity affected the composition of bacteria. 


\section{ACKNOWLEDGEMENTS}

This study was supported by Grant RTA Universitas Gadjah Mada, Indonesia No. 2129/UN1/DITLIT/DITLIT/LT/2019. The authors are grateful to the farmers who have allowed researchers to collect samples and data to conduct this study.

\section{REFERENCES}

Abia ALK, Alisoltani A, Keshri J, Ubomba-Jaswa E. 2018. Metagenomic analysis of the bacterial communities and their functional profiles in water and sediments of the Apies River, South Africa, as a function of land use. Sci Total Environ 616-617: 326-334. DOI: 10.1016/j.scitotenv.2017.10.322.

Bentzon-Tilia M, Sonnenschein EC, Gram L. 2016. Monitoring and managing microbes in aquaculture-towards a sustainable industry. Microb Biotechnol 9 (5): 576-84.

Caporaso JG, Kuczynski J, Stombaugh J et al. 2010. QIIME allows analysis of high-throughput community sequencing data Intensity normalization improves color calling in SOLiD sequencing. Nature 7 (5): 335-336. DOI: 10.1038/nmeth0510-335

Dabadé DS, Wolkers-Rooijackers JCM, Azokpota P et al. 2016. Bacterial concentration and diversity in fresh tropical shrimps (Penaeus notialis) and the surrounding brackish waters and sediment. Intl J Food Microbiol 218: 96-104. DOI: 10.1016/j.ijfoodmicro.2015.11.013.

Dittmann KK, Rasmussen BB, Castex M, Gram L, Bentzon-Tilia M 2017. The aquaculture microbiome at the centre of business creation. Microb Biotechnol 10 (6): 1279-1282.

Duarte LN, Coelho FJRC, Cleary DFR, Bonifácio D, Martins P, Gomes NCM. 2019. Bacterial and microeukaryotic plankton communities in a semi-intensive aquaculture system of sea bass (Dicentrarchus labrax): A Seasonal Survey. Aquaculture 503: 59-69. DOI: 10.1016/j.aquaculture.2018.12.066.

Edgar RC. 2004. MUSCLE: Multiple sequence alignment with high accuracy and high throughput. Nucleic Acids Res 32 (5): 1792-1797. DOI: $10.1093 /$ nar/gkh340.

Edgar RC. 2010. Search and clustering orders of magnitude faster than BLAST. Bioinformatics 26 (19): 2460-2461. DOI: 10.1093/bioinformatics/btq461.

Edgar RC, Haas BJ, Clemente JC, Quince C, Knight R. 2011. UCHIME improves sensitivity and speed of chimera detection. Bioinformatics 27 (16): 2194-2200. DOI: 10.1093/bioinformatics/btr381.

Fan L, Chen J, Meng S, Song C, Qiu L, Hu G, Xu P. 2015. Characterization of microbial communities in intensive GIFT tilapia (Oreochromis niloticus) pond systems during the peak period of breeding. Aquacult Res 48 (2): 459-472. DOI: 10.1111/are.12894.

FAO. 2020. The State of World Fisheries and Aquaculture 2020. In Food and Agriculture Organization of the United Nations, Fisheries Department, Rome, Italy.

Fernandes SO, Kirchman DL, Michotey VD, Bonin PC, Lokabharathi PA. 2014. Bacterial diversity in relatively pristine and anthropogenicallyinfluenced mangrove ecosystems (Goa, India). Braz J Microbiol 4 5(4): 1161-1171.

Gupta RS. 2000. The phylogeny of proteobacteria: Relationships to other eubacterial phyla and eukaryotes. FEMS Microbiol Rev 24 (4): $367-$ 402. DOI: 10.1016/S0168-6445(00)00031-0.

Hahn MW. 2006. The microbial diversity of inland waters. Curr Opin Biotechnol 17: 256-261. DOI: 10.1016/j.copbio.2006.05.006.

Herlambang A., Murwantoko, Istiqomah I. 2021. Dynamic change in bacterial communities in the integrated rice-fish farming system in Sleman, Yogyakarta, Indonesia. Aquacult Res. DOI: 10.1111/are.15432.
Hornick KM, Buschmann AH. 2018. insights into the diversity and metabolic function of bacterial communities in sediments from Chilean Salmon Aquaculture Sites. Ann Microbiol 68 (2): 63-77. DOI: $10.1007 / \mathrm{s} 13213-017-1317-8$.

Huang W, Chen X, Jiang X, Zheng B. 2017. Characterization of sediment bacterial communities in plain lakes with different trophic statuses. Microbiol Open 6 (5): e00503. DOI: 10.1002/mbo3.503.

Ihaka R, Gentleman R. 1996. R: A Language for Data Analysis and Graphics. J Comput Graph Stat 5 (3): 299-314. DOI: 10.1080/10618600.1996.10474713.

Kersters K, De Vos P, Gillis M, Swings J, Vandamme P, Stackebrandt E. 2006. Introduction to the Proteobacteria. In: Dworkin M, Falkow S, Rosenberg E, Schleifer K, Stackebrandt E (eds). The Prokaryotes. Springer, Nederland. DOI: 10.1007/0-387-30745-1_1.

Kurilkina M I, Zakharova YR, Galachyants YP, et al. 2016. Bacterial community composition in the water column of the deepest freshwater Lake Baikal as determined by next-generation sequencing. FEMS Microbiol Ecol 92 (7): fiw094. DOI: 10.1093/femsec/fiw094.

Li D, Yang M, Li Z, Qi R, He J, Liu H. 2008. Change of bacterial communities in sediments along Songhua River in Northeastern China after a nitrobenzene pollution event. FEMS Microbiol Ecol 65 (3): 494-503. DOI: 10.1111/j.1574-6941.2008.00540.x

Martin M. 2011. Cutadapt removes adapter sequences from highthroughput sequencing reads. Embnet J 17 (1): 10-12.

Martins P, Coelho FJRC, Cleary DFR et al. 2018. Seasonal patterns of bacterioplankton composition in a semi-intensive European seabass (Dicentrarchus labrax) aquaculture system. Aquaculture 490 (February): 240-50. DOI: 10.1016/j.aquaculture.2018.02.038.

Newton RJ, Jones SE, Eiler A, Mcmahon KD, Bertilsson S. 2011. A guide to the natural history of freshwater lake bacteria. Microbiol Mol Biol Rev 75 (1) :14-49. DOI: 10.1128/MMBR.00028-10.

Newton RJ, Kent AD, Triplett EW, Mcmahon KD. 2006. Microbial community dynamics in a humic lake: differential persistence of common freshwater phylotypes. Environ Microbiol 8 (6): 956-970. DOI: 10.1111/j.1462-2920.2005.00979.x.

Qin Y, Hou J, Deng M, Liu Q, Wu C, Ji Y, He X. 2016. Bacterial abundance and diversity in pond water supplied with different feeds. Sci Rep 6: 35232. DOI: 10.1038/srep35232.

Quast C, Pruesse E, Yilmaz P, Gerken J, Schweer T, Yarza P, Peplies J, Glöckner FO. 2013. The SILVA ribosomal RNA gene database project: Improved data processing and web-based tools. Nucleic Acids Res 41 (D1): 590-596. DOI: 10.1093/nar/gks1219.

Roquigny R, Mougin J, Le Bris C, Bonnin-Jusserand M, Doyen P, Grard T. 2021. Characterization of the marine aquaculture microbiome: a seasonal survey in a seabass farm. Aquaculture 531 (September 2020): 735987. DOI: 10.1016/j.aquaculture.2020.735987.

Sharmin F, Wakelin S, Huygens F, Hargreaves M. 2013. Firmicutes dominate the bacterial taxa within sugar-cane processing plants. Sci Rep 3: 3107. DOI: 10.1038/srep03107.

Wang Z, Li W, Li H, Zheng W, Guo F. 2020. Phylogenomics of Rhodocyclales and Its Distribution in Wastewater Treatment Systems. Sci Rep 10 (1): 1-12. DOI: 10.1038/s41598-020-60723-X.

Yu Y, Lee C, Kim J, Hwang S. 2005. Group-specific primer and probe set to detect methanogenic communities using quantitative real-time polymerase chain reaction. Biotechnol Bioeng 89 (6): 670-679. DOI: 10.1002/bit.20347.

Zhang L, Zhao T, Wang Q, Li L, Shen T, Gao G. 2019. Bacterial community composition in aquatic and sediment samples with spatiotemporal dynamics in large, shallow, eutrophic Lake Chaohu, China. J Freshwater Ecol 34 (1): 575-589. DOI: 10.1080/02705060.2019.1635536.

Zhao D, Cao X, Huang R, Zeng J, Wu QL. 2017. Variation of bacterial communities in water and sediments during the decomposition of microcystis biomass. PLoS ONE 12 (4): e0176397. DOI: 10.1371/journal.pone.0176397. 